\title{
High-Throughput Manufacture of 3D Fiber Scaffolds for Regenerative Medicine
}

\author{
Rohan A. Shirwaiker, $\mathrm{PhD},{ }^{1-3,{ }^{*}}$ Matthew B. Fisher, $\mathrm{PhD},{ }^{2,3,{ }^{*}}$ Bruce Anderson, ${ }^{4}$ \\ Karl G. Schuchard, ${ }^{1,3}$ Paul B. Warren, PhD, ${ }^{2,3}$ Benoit Maze, PhD, ${ }^{4}$ Pierre Grondin, ${ }^{4}$ \\ Frances S. Ligler, DPhil, DSc, ${ }^{2-4}$ and Behnam Pourdeyhimi, PhD ${ }^{3,4}$
}

Engineered scaffolds used to regenerate mammalian tissues should recapitulate the underlying fibrous architecture of native tissue to achieve comparable function. Current fibrous scaffold fabrication processes, such as electrospinning and three-dimensional (3D) printing, possess application-specific advantages, but they are limited either by achievable fiber sizes and pore resolution, processing efficiency, or architectural control in three dimensions. As such, a gap exists in efficiently producing clinically relevant, anatomically sized scaffolds comprising fibers in the $1-100 \mu \mathrm{m}$ range that are highly organized. This study introduces a new highthroughput, additive fibrous scaffold fabrication process, designated in this study as 3D melt blowing (3DMB). The 3DMB system described in this study is modified from larger nonwovens manufacturing machinery to accommodate the lower volume, high-cost polymers used for tissue engineering and implantable biomedical devices and has a fiber collection component that uses adaptable robotics to create scaffolds with predetermined geometries. The fundamental process principles, system design, and key parameters are described, and two examples of the capabilities to create scaffolds for biomedical engineering applications are demonstrated.

Keywords: 3D printing, melt blowing, fibers, scaffolds

\section{Impact Statement}

Three-dimensional melt blowing (3DMB) is a new, high-throughput, additive manufacturing process to produce scaffolds composed of highly organized fibers in the anatomically relevant $1-100 \mu \mathrm{m}$ range. Unlike conventional melt-blowing systems, the 3DMB process is configured for efficient use with the relatively expensive polymers necessary for biomedical applications, decreasing the required amounts of material for processing while achieving high throughputs compared with $3 \mathrm{D}$ printing or electrospinning. The $3 \mathrm{DMB}$ is demonstrated to make scaffolds composed of multiple fiber materials and organized into complex shapes, including those typical of human body parts.

\section{Introduction}

$\mathbf{E}$ NGINEERING LIVING TISSUES require recreation of both the three-dimensional (3D) organization of cells as well as extracellular features of the tissue to support the required tissue functions. ${ }^{1,2}$ The unique requirements of scaffolds used to mimic the extracellular matrix (ECM) are evident when comparing the diversity of fibrous ECM within the multitude of human body parts, including nerve bundles, ${ }^{3}$ blood vessels, ${ }^{4}$ skin, ${ }^{5}$ lungs, ${ }^{6}$ intestines, ${ }^{7}$ kidneys, ${ }^{8}$ mus- cles, ${ }^{9,10}$ and joints. ${ }^{11}$ In all of these tissues, the unique size, orientation, and chemistry of the underlying fibrous matrix provide structural stability, influence molecular transport, and organize multiple cell types to achieve a specific function. ${ }^{12,13}$ Recapitulating these fibrous, tissue-specific ECM structures is a critical challenge.

Over decades, processes, such as phase separation, ${ }^{3,14,15}$ particle leaching, ${ }^{16,17}$ gas foaming, ${ }^{18-20}$ and emulsification, ${ }^{21}$ have been used to create porous scaffolds. However, these processes are not ideal for creating fibrous structures

\footnotetext{
${ }^{1}$ Edward P. Fitts Department of Industrial and Systems Engineering, North Carolina State University, Raleigh, North Carolina, USA.

${ }^{2}$ Joint Department of Biomedical Engineering, University of North Carolina at Chapel Hill and North Carolina State University, Raleigh, North Carolina, USA.

${ }^{3}$ Comparative Medicine Institute, ${ }^{4}$ The Nonwovens Institute, North Carolina State University, Raleigh, North Carolina, USA.

*These authors contributed equally to this work.

(C) Rohan A. Shirwaiker, et al. 2020; Published by Mary Ann Liebert, Inc. This Open Access article is distributed under the terms of the Creative Commons Attribution Noncommercial License (http://creativecommons.org/licenses/by-nc/4.0/) which permits any noncommercial use, distribution, and reproduction in any medium, provided the original author(s) and the source are cited.
} 
necessary to replicate several tissue types (e.g., musculoskeletal, neural) wherein the fiber organization is key to the tissue function and biomechanics. Typical strategies for creating fibrous synthetic scaffolds have focused on two broader categories of processes-electrostatic fiber formation $(\mathrm{EFF})^{22,23}$ and 3D printing ${ }^{10,24-26}$-with fundamentally different fiber formation and collection principles.

Electrospinning is the most widely used EFF process in which fiber formation is achieved through electrostatic repulsion forces between charged surfaces causing the continuous emission of fibers from a viscous fluid. The fibers are collected on a stationary substrate or a rotating collector to constitute the scaffold. ${ }^{9}$ Based on process parameters, such as applied voltage, needle diameter, needle-to-collector distance, solvent concentration, and solvent viscosity, fibers can be achieved with diameters in the range of $50 \mathrm{~nm}$ to several microns. ${ }^{4,9,20,22,23,27}$ However, creating scaffolds with complex anatomical geometries using electrospinning systems is challenging, and these systems have struggled to produce scaffolds more than $1 \mathrm{~mm}$ thick-many tissues are much thicker. ${ }^{25}$ For certain polymers, the need for organic solvents in these processes also impacts the choice of starting materials and necessitates careful precleaning of scaffolds before use with cells.

In comparison, 3D printing (3DP) processes excel at reproducibly fabricating scaffolds with patient-specific macrogeometry. In extrusion-based 3DP, the most commonly used scaffold fabrication principle, fibers are formed through pneumatic extrusion of a viscoelastic fluid (e.g., molten polymers, hydrogel solutions) onto a substrate through a syringe needle that traverses a computer-aided toolpath one layer at a time. The ability of these processes to achieve fibrous architectures with fine structures recapitulating native ECM is limited. Depending on parameters, such as extrusion pressure, syringe temperature, and needle size, the achievable fiber diameters typically range in the upper tens to hundreds of microns. ${ }^{28}$

Recently, electrohydrodynamic printing and electrowriting, which integrate principles from EFF (fiber formation modality) and 3D printing (computer-aided tool path), have also been investigated. $^{28,29}$ Although this approach provides good spatial control over fibers similar in diameter to electrospinning, the process is not efficient in creating scaffolds of clinically relevant thickness. As such, there is a need to develop more efficient biofabrication processes to create scaffolds that can recapitulate the micro- and macrostructure of native tissues.

The 3D melt-blowing (3DMB) process introduced in this work enables the creation of fibrous scaffolds with anatomically relevant macrogeometry and thickness while mimicking ECM architecture with fiber diameters in the range of $1-100 \mu \mathrm{m} .{ }^{30-32}$ The process has demonstrated the capacity to produce biocompatible fibers with predefined sizes and porosity, combinations of fibers with different chemical and physical properties, and fiber deposition with orientation controlled in three dimensions. The 3DMB offers significantly higher processing efficiency than EFF and 3DP-based processes. The typical polymer throughput in $3 \mathrm{DMB}$ is in the order of $100 \mathrm{mg} /$ needle/min compared with $5 \mathrm{mg} /$ needle/ min for electrospinning. ${ }^{33}$ For comparison, it would take $\sim 8 \mathrm{~h}$ to $3 \mathrm{D}$ print a human polycaprolactone (PCL) scaffold for replacement of the knee meniscus through the extrusionbased 3D bioplotting process with fiber diameters and pores on the order of $100-200 \mu \mathrm{m}$, but the 3DMB process de- scribed in this study created a meniscus scaffold in under 15 min. ${ }^{34}$ The 3 DMB represents a scalable, high-throughput technology capable of producing clinically translatable scaffolds in a few minutes.

This article describes the 3DMB operational principles, device design, and examples of fibers created from a variety of biocompatible polymers to demonstrate control of size, orientation, and physical properties. Two examples of scaffolds with unique designs, difficult if not impossible to fabricate using other methods, are also presented: a device for hernia repair that includes both stiff and stretchy fibers with a unique physical organization and maneuverability, and a knee meniscus scaffold with complex micro- and macroscale anatomical features.

\section{Methods}

The 3DMB system combines melt-blown fiber formation and a variety of computer-aided fiber collection mechanisms (Fig. 1). The interaction of these primary components is critical to the development of fibrous scaffolds to recapitulate the desired micro- and macroarchitectures of tissues. Key components of the system for scaffold production are the stationary melt-blowing die, through which polymer fibers are extruded and attenuated by high-pressure heated air flow and the collector underneath.

Fibers are formed using traditional strategies for melt blowing of polymers, ${ }^{35-40}$ but with alterations for biomedical applications (Fig. 1A). Polymer is first fed through a hopper, which is smaller than that used in traditional meltblowing systems and ideal for expensive biomedical-grade polymers. Polymer is then heated and extruded, and the flow rate of the polymer is controlled through an applied pressure. The polymer is then directed through a die. The die consists of an array of nozzles ( $5 \times 5$ with $2 \mathrm{~mm}$ center-tocenter spacing), each allowing interaction of polymer with coaxial air flow. Specifically, the die has $3 \times 3$ nozzles for polymer and coaxial air with an additional row around the perimeter with air only (air curtain). This configuration is beneficial in terms of fiber formation and the evolution of the morphology in the fiber structure. The edge-to-edge distance between adjacent coaxial nozzles is $0.65 \mathrm{~mm}$, which minimizes crosscurrent effects of adjacent air and polymer streams. In contrast to conventional dies comprising many nozzles in a single row, this die configuration utilizes multiple rows of fewer nozzles to produce high throughput of well-shaped fibers. In each nozzle, fiber formation is achieved as the air drawing forces overcome the polymer rheological force. Here, the interaction of the coaxial air jet with the viscoelastic polymer emerging from the nozzle under high pressure causes the polymer stream to stretch.

Different collection mechanisms can be configured to achieve the final desired scaffold design (Fig. 1B). Of high relevance to biomedical applications, the collector can be biomodeled to patient-specific geometry using medical images and 3D printed out of thermally resistant materials, such as acrylonitrile butadiene styrene (ABS). The collector is loaded onto a six-axis robot arm and positioned underneath the melt-blowing die. The collector can be translated along the six-axes or rotated through a motor-driven shaft at different linear and rotational speeds to control the deposition characteristics of the fibers. The interaction of the 

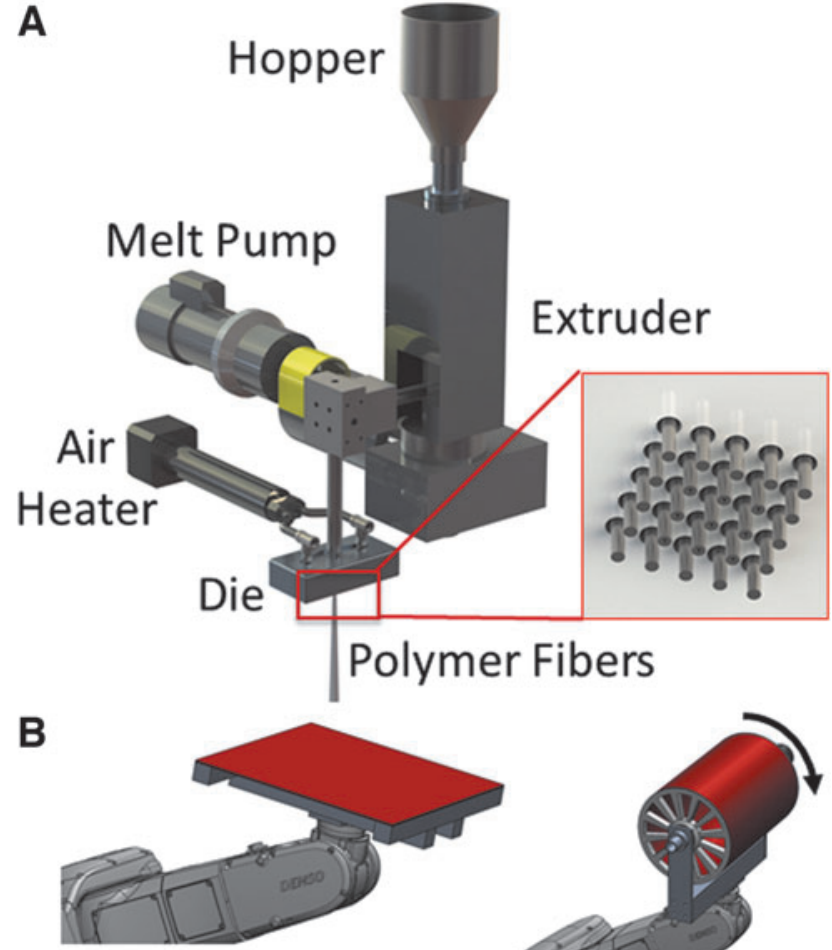

Flat Collector

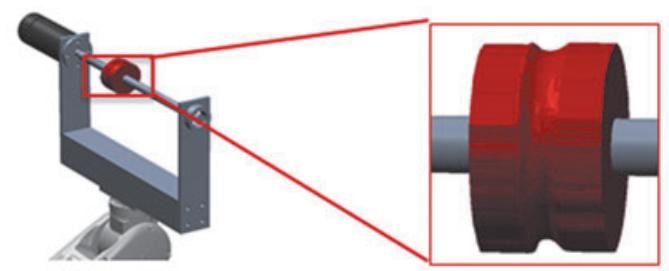

Negative Mold Collector

FIG. 1. The 3DMB System. The system has two major sections. (A) In the fiber melt-blowing portion, an extruder converts solid pellets into molten polymer, a melt pump accurately meters the polymer flow through small nozzles in the die to form fibers, and an air heater (along with air flow) attenuates the fibers and manages the temperature of the formed fibers. (B) In the collection system, a collector adapted to create the desired scaffold geometry is mounted on a robotic arm and controls the location, speed, and angle of the fiber deposition. 3DMB, three-dimensional melt blowing.

collector with the melt-blown fiber is essential to the 3DMB process-the collector not only acts as a substrate or mold onto which the melt-blown fibers are deposited to create the desired 3D geometry, but its position and motion with respect to the die also impact the fiber morphology and orientation. The fibers experience a bending instability as they emit from the die, causing them to whip and buckle. Matching the collector surface speed to fiber speed eliminates the bending instability and the rotation of the collector pulls the fibers into alignment along the direction of the collector motion. Speeds of up to $15 \times 10^{5} \mathrm{~mm} / \mathrm{min}$ could be generated at the surface of the collector in this system.

Several processing parameters, including chemistry of the polymer, the melt-blowing conditions, collection mechanism, and time of collection, together govern the macro- geometry and microarchitecture of the fibrous structure. Parameters investigated in this study include variations in the polymer, polymer flow rate (controlled by polymer temperature and extruder pressure), air temperature, air flow rate, die-collector distance (DCD, the distance between the die face and deposition location on the collector), surface velocity of collection (SVC), fiber deposition offset (FDO, the lateral distance between the collector's central axis and the position of the central nozzle), angle between collection surface and polymer stream, and transverse collection velocity.

\section{Experiments}

Experimental design at a glance. The 3DMB system was constructed to produce fibrous scaffolds with oriented depositions in three dimensions. A design of experiments strategy was employed to determine the operating parameters to produce fibers of defined diameter and organization out of multiple materials. Finally, multicomponent scaffolds and scaffolds with complex 3D geometry predesigned to mimic a human tissue geometry were demonstrated to exemplify the type of architectures possible with the 3DMB approach. The following methods detail the device components, the variety of materials used, the range of diameters produced, and the flexibility in the types of scaffolds possible. Analytical methods used to characterize the fibers and scaffolds are described.

Details of the 3D melt blowing system. The first step in the process of producing the melt-blown fibers is to melt the polymer pellets in an extruder. A Randcastle 0625 vertical extruder with a 24:1 L/D mixing screw (Randcastle Extrusion Systems, Inc., NJ) was used for the system. A closedloop coolant chiller is used to cool both feed-throat and gearbox. From the extruder, a set of transfer zones are used to move the molten polymer from the output point of the extruder to the $3 \times 3$ nozzle Biax melt-blown die (Biax, FiberFilm, WI). Throughput is controlled through pressure feedback with the 3-phase motor of the extruder and viscosity (temperature) control of the polymer temperature.

The Biax die placed at the end of extruder has nine active polymer nozzles arranged in a $3 \times 3$ matrix. An additional row of "dead" nozzles is located around the perimeter serving as an air curtain. Heated air is routed through the die block and each nozzle has its own concentric air jet. Airflow control is managed with a mass flow controller (Vögtlin Instruments, Switzerland) with a max flow rate of $450 \mathrm{~L} /$ min. An HA2-12 air process heater (Backer Hotwatt, MA) is used to provide consistent hot air to the melt-blown die.

Samples are produced using a variety of collection systems. The extruder and die are stationary, while the collectors are small enough to be mounted on an XYZ stage or a robot arm with $6^{\circ}$ of freedom of motion. Collection surfaces were mounted on either a Benchtop PRO 2424 XYZ stage (Avid CNC, WA) with 24 in $\times 24$ in working area or a Denso VS68 robot arm (Denso, Japan). Collection surfaces included a flat surface (up to 24 in $\times 24$ in on the Benchtop PRO and 12 in $\times 12$ in on the Denso Robot), a rotating drum capable of surface speeds in excess of $15 \times 10^{5} \mathrm{~mm} / \mathrm{min}$ (diameter ranges from 6 to 8 in) and a small rotating mandrel mount that was used for smaller diameter rods 
(1/4-1 in) as well as the collector mold specially designed to produce the meniscus shape.

The system is controlled through a human-machine interface that manages signals from the heating, drive, and robot systems. Programming of the robot or stage is done with vendor software (Mach 3 for the stage and WINCAPS III for the Denso). Communication through the programming software can enable and set process variables dynamically during sample collection.

Assessing effects of 3DMB process parameters on properties of fibrous PCL scaffolds. Effects of three important 3DMB parameters-SVC $\left(1.5 \times 10^{5} \mathrm{~mm} / \mathrm{min}, 7 \times 10^{5} \mathrm{~mm} /\right.$ $\mathrm{min})$, FDO (0 $\mathrm{mm}$ [at the central axis], $80 \mathrm{~mm}$ [offset toward the incoming edge]), and DCD $(150 \mathrm{~mm}, 200 \mathrm{~mm})$-on three scaffold characteristics-fiber diameter, fiber angular coherency, and tensile elastic modulus-were assessed. These process parameter levels represent the limits within which 3D fibrous PCL scaffolds could be feasibly fabricated. Molten PCL (Mw $43 \mathrm{kDa}$, Polysciences, PA) at $130^{\circ} \mathrm{C}$ was extruded through the die with nine nozzles $(\varnothing 300 \mu \mathrm{m}$ each) at a polymer throughput of $100 \mathrm{mg} / \mathrm{nozzle} / \mathrm{min}$. The polymer streams were attenuated with air at a temperature of $140^{\circ} \mathrm{C}$ air and total throughput of $110 \mathrm{~L} / \mathrm{min}$. The resulting fibers were collected onto a rotating metal mandrel $(\varnothing 208 \mathrm{~mm})$ covered with poly-coated paper (Gordon Paper Company, Virginia Beach, VA) to obtain 3-mm thick melt-blown samples at each parameter combination. The poly-coated paper aided in the removal of the fibrous sample postfabrication.

Scanning electron microscopy (SEM; Phenom Pro, Phenom, Netherlands) micrographs of melt-blown samples ( $n=3$ per parameter combination) were sputter coated with gold-palladium (60:40) for $90 \mathrm{~s}$, at $29 \mathrm{~mA}$ DC and 0.05 Torr, imaged at $5 \mathrm{kV}$ accelerating voltage, and analyzed using ImageJ and a custom MATLAB script to determine the mean fiber diameter. Furthermore, the OrientationJ plugin was used to characterize the angular coherency of fibers in each image. Angular coherency is a metric of anisotropy based on the analyses of the structure tensor of the image. This structure tensor is a matrix derived from the image, evaluated at each pixel. The structure tensor carries essential information about the orientation direction and intensity of the edges of the fibers within the image. The ratio of the degree of orientation to the edge intensity at each pixel yields the local angular coherency. The angular coherency of the entire image was derived from the distribution of aforementioned local angular coherencies, on a scale of $0 \%$ to $100 \%$. Greater angular coherency indicates a higher proportion of fibers oriented along a principal direction in the image.

For analysis of mechanical properties, dog-bone specimens ( $n=3$ per parameter combination), as per ASTM D1708-18 (gage dimensions: $5 \times 22 \mathrm{~mm}$ ), were punched from meltblown samples along the direction of collector rotation. The samples were tensile tested using a universal testing system (5944; Instron) to failure with a $500 \mathrm{~N}$ load cell at a uniaxial displacement rate of $10 \mathrm{~mm} / \mathrm{min}$. The elastic region of each stress-strain curve was automatically identified, and tensile modulus was then calculated. Tensile strength was normalized to the sample weight.

Based on the observed fibrous characteristics, samples of two groups with contrasting fiber-pore morphologies fabri- cated with SVC/FDO/DCD of $1.5 \times 10^{5} / 0 / 150$ and $7 \times 10^{5} /$ $80 / 150$ were evaluated for the ability to support cell culture as check for short-term biocompatibility. In addition to fiber diameter and angular coherency, scaffold porosity of the two groups was determined in ImageJ wherein the fibers in the foreground of SEM micrographs $(n=3 /$ sample) were segmented from the background, their area calculated, and then subtracted from the total micrograph area. Disc-shaped scaffolds $(\varnothing 8 \mathrm{~mm})$ were sectioned from samples in both groups. The scaffolds were treated with $2.5 \mathrm{M}$ sodium hydroxide for $1 \mathrm{~h}$ to improve their hydrophilicity. They were subsequently sterilized by soaking in $70 \%$ ethanol for $30 \mathrm{~min}$ and rinsing thoroughly with sterile phosphatebuffered saline. Cell seeding was performed in a 48-well plate using a multistep procedure. First, $2.5 \times 10^{5} \mathrm{NIH} 3 \mathrm{~T} 3$ cells were seeded onto one side and cultured in Eagle's minimum essential medium supplemented with $10 \%$ fetal bovine serum over $24 \mathrm{~h}$. Then, the scaffolds were inverted, seeded with $2.5 \times 10^{5}$ cells on the opposite side, and cultured in the medium for another $24 \mathrm{~h}$. At this time (Day 1), one sample of each morphology was assessed for cell viability using Live/Dead assay (Thermo Fisher Scientific, MA). In parallel, three samples of each morphology were cultured in the medium for 7 days and cellular metabolic activity was assessed through alamarBlue assay (Thermo Fisher Scientific) at days 2 and 7.

Fabrication of scaffold: example 1 featuring two different kinds of fibers. The hernia plug consisted of two layers of melt-blown fibers, one layer made of PCL (Polysciences) and the other layer of 6260D PLA (NatureWorks, MN). Samples were made using a rotating drum attached to a robotic arm with 6 degrees of freedom of motion (Denso VS68). The fibers were highly oriented in such a manner as to allow the web to be stretched in the crossdirection. The stretch enabled the web to be turned into a tube (bonding the edges) and subsequently rolled into a plug. The complete web was about $100 \mathrm{~g} / \mathrm{m}^{2}$ with similar proportions of PCL and polylactic acid (PLA).

The PCL web (50 gsm web, deposited using $0.08 \mathrm{ghm}$ polymer) was fabricated using low-volume air flow $(35 \mathrm{~L} /$ min) and matching SVC to filament spinning speed $(50 \mathrm{~m} /$ min). These settings created a highly oriented web with two distinct orientations. The angle between the fibers was controlled by changing the velocity of the traverse motion ( $25 \mathrm{~m} / \mathrm{min}$ ). The optimum web created for the purpose of the hernia plug had an angle of $\sim 60^{\circ}$ between fibers.

Once the PCL webs were fabricated, they were cut in half and secured to the drum using painter's tape. PLA 6260D was then processed (40 gsm PLA web, deposited using 0.25 ghm polymer, $175 \mathrm{~L} / \mathrm{min}$ air volume) into fibers $5-10 \mu \mathrm{m}$ in diameter that were highly oriented $(75 \mathrm{~mm}$ positive offset). The PLA was sprayed directly onto the PCL webs at a collection velocity of $200 \mathrm{~m} / \mathrm{min}$, and the heat from the PLA fibers used to bind them to the PCL. The two-layer PCL/ PLA web was then rolled and bonded using a heated knife edge to the desired diameter.

Fabrication of scaffold: example 2 featuring complex 3D architecture. A stereolithography (STL) model of medial knee meniscus reconstructed from magnetic resonance imaging (MRI) scan of a human knee ${ }^{24}$ was processed in 
Autodesk Meshmixer using Boolean operations to form a mold cavity with net shape of the meniscus model on a cylindrical mandrel. The cavity was smoothened to match the meniscus' depth and width to allow for uninterrupted melt-blown fiber collection during rotation. This final model of the cylindrical mandrel with the meniscus-shaped cavity was then 3D-printed out of ABS on a uPrint system (Stratasys, MN). PCL melt blown through the die with three nozzles $\left(\varnothing 300 \mu \mathrm{m}\right.$ each) at $150^{\circ} \mathrm{C}$ with a polymer throughput of $0.15 \mathrm{ghm}$ and attenuating air temperature and throughput of $150^{\circ} \mathrm{C}$ and $50 \mathrm{~L} / \mathrm{min}$, respectively, was collected onto the biomodeled mandrel rotating at $600 \mathrm{rpm}$.

\section{Results and Discussion}

Relationship between 3DMB process parameters and scaffold characteristics. The levels of the three key 3DMB parameters, SVC, DCD, and FDO, in Table 1 are the extreme conditions attainable with the system when using the rotating collector mechanism in Figure 1B with a radius of $\sim 104 \mathrm{~mm}$. The FDO parameter can, with some fiber materials, extend as far as the radius of the collector. With other materials, the FDO is limited in practice to $\sim 80 \%$ of the collector radius due to the stochastic nature of the fiber movement through space until it is collected. DCD is similarly limited by the fiber trajectory, which becomes more dispersed as the distance from the die to the collector grows. $\mathrm{SVC}$ is limited by the maximal rotational speed and torque of the collector motor. The range of SVC, DCD, and FDO levels at which fibrous scaffolds can be reproducibly achieved vary by the polymer.

Figure 2 highlights how changes in DCD, SVC, and FDO affect the fibrous properties of the PCL scaffolds (Fig. 1B). For this parameter space, viable scaffolds could be produced at all parameter combinations, except at higher SVC and DCD values and lower FDO values (Fig. 2B). Among viable groups, mean fiber diameters ranged between $4.4-8.1 \mu \mathrm{m}$, angular coherency between $8 \%$ and $85 \%$ (coherency defined as the proportion of fibers aligned along the direction of rotation of the collector), and tensile strength to weight ratio between 7.1 and $16 \mathrm{MPa} / \mathrm{g}$. Different parameter combinations were able to produce fiber ensembles with obvious differences in morphology (Fig. 2B), which were further quantified (Fig. 2C-E). Scaffolds fabricated at lower SVC, FDO, and DCD values demonstrated the largest fiber diameter and lowest angular coherency. Increasing the SVC and FDO resulted in a 50\% decrease in the scaffold fiber diameter and $1300 \%$ increase in angular coherency. But, the effects of changes in DCD on fiber morphology were not appreciable (generally $<10 \%$ ). Furthermore, the tensile strength-to-weight ratio correlated well with the scaffolds' angular coherency. Scaffolds fabricated at the higher FDO and SVC had higher angular coherency of the fibers as well as $77 \%$ greater tensile strength-to-weight ratio. This implies that scaffolds with a higher proportion of fibers aligned along the principal direction of rotation of the $3 \mathrm{DMB}$ collector possessed higher tensile strength along that direction, as hypothesized.

Process parameters also influenced cellular interactions. As a preliminary check on biocompatibility, scaffolds featuring different fiber morphologies were cultured with $\mathrm{NIH}$ 3 T3 fibroblasts (Fig. 3). Specifically, we compared scaffolds featuring high fiber diameter, low angular coherency, and lower porosity $\left(\mathrm{SVC} / \mathrm{FDO} / \mathrm{DCD}\right.$ of $\left.1.5 \times 10^{5} / 0 / 150\right)$ with scaffolds featuring low fiber diameter, high angular coherency, and higher porosity (SVC/FDO/DCD of $7 \times 10^{5} / 80$ / $150)$. Differences were also observed in the biological characteristics of groups that possessed the greatest contrast in fiber morphology-SVC/FDO/DCD of $1.5 \times 10^{5} / 0 / 150$ (highest fiber diameter, lowest angular coherency, 28.7\% \pm $1.25 \%$ porosity) and $7 \times 10^{5} / 80 / 150$ (lowest fiber diameter, highest angular coherency, $42.1 \% \pm 0.44 \%$ porosity). Scaffolds were cultured with NIH 3 T3 fibroblasts. At day 1, the scaffolds of the former group supported cells with higher viability. In contrast, at days 2 and 7, scaffolds of the more porous, more aligned group demonstrated higher cellular metabolic activity.

These results highlight the interplay between $3 \mathrm{DMB}$ process parameters and how the parameters can be modulated to tune the scaffold morphology, mechanical properties, and biocompatibility. The 3DMB process can be optimized to engineer scaffolds with properties to match the tissue application by appropriately tuning and controlling these parameters.

Examples of fiber production and alignment. The 3DMB system offers versatility in the polymers that can be used. Figure 4 demonstrates fibrous scaffolds fabricated from a variety of thermoplastic materials, including polyesters, polyurethanes, and polyester elastomers under the conditions listed in Supplementary Table S1. For each material, the process could be tuned to produce reproducible scaffolds comprising uniform micron-diameter fibers. Interestingly, morphology was dependent on material class, with polyester fibers appearing straight, while fibers made from polyurethane or polyester elastomer had higher tortuosity.

The 3DMB system provided excellent control over fiber diameter through the numerous tunable processing parameters. To illustrate this concept, several fiber scaffolds

Table 1. Range of Process Parameters Achievable with the Three-Dimensional Melt-Blowing System

\begin{tabular}{|c|c|c|c|c|}
\hline Parameter & Unit & & Minimum & Махітит \\
\hline Polymer temperature & $\mathrm{C}$ & & 130 & 300 \\
\hline Polymer throughput & ghm & $\operatorname{gram} /($ hole*minute $)$ & 0.05 & 1 \\
\hline Cold air volume & $1 / \mathrm{min}$ & liters/minute & 25 & 300 \\
\hline Die collector distance & $\mathrm{mm}$ & millimeters & 10 & 400 \\
\hline Surface velocity of collection & $\mathrm{m} / \mathrm{min}$ & meters/minute & 1 & 1500 \\
\hline Traverse collection velocity & $\mathrm{m} / \mathrm{min}$ & (collector movement) & 0 & 60 \\
\hline Fiber deposition offset (for a radius of collection of $104 \mathrm{~mm}$ ) & $\mathrm{mm}$ & & 0 & \pm 100 \\
\hline Passes & $\#$ & number of "layers", & 1 & No limit \\
\hline
\end{tabular}



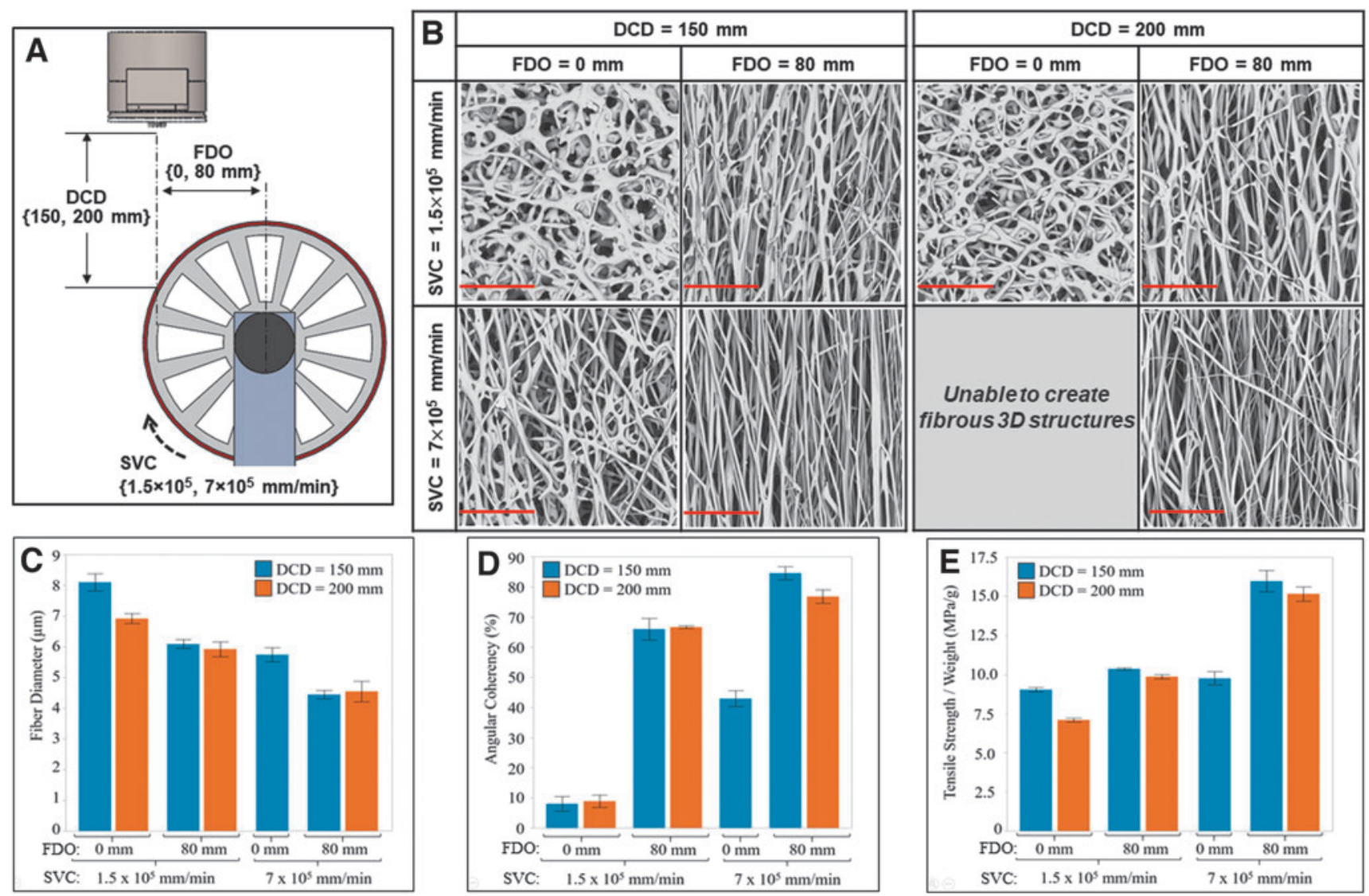

FIG. 2. Control of PCL Fiber Production: Effects of three key 3DMB process parameters-(A) SVC, DCD, and FDL-on $(\mathbf{B}, \mathbf{C})$ produced fiber diameter, (B, D) angular coherency, and (E) tensile strength/weight ratio. DCD, die-collector distance; FDL; PCL, polycaprolactone; SVC, surface velocity of collection.

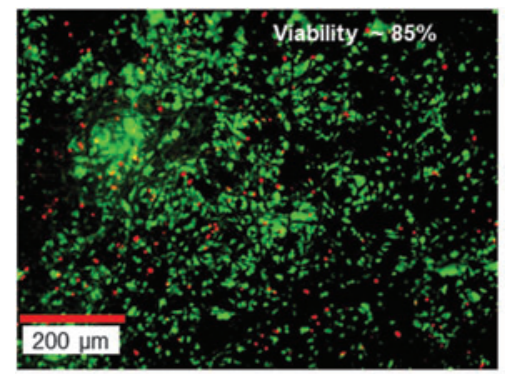

$\mathrm{DCD}=150 \mathrm{~mm}$ FDO $=0 \mathrm{~mm} \mathrm{SVC}=1.5 \times 10^{5} \mathrm{~mm} / \mathrm{min}$ Angular Coherency: $8 \%$

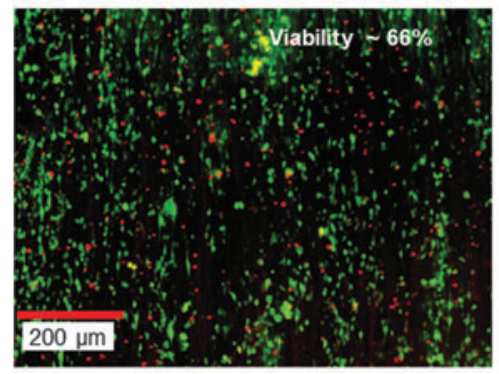

$\mathrm{DCD}=150 \mathrm{~mm}$ FDO $=80 \mathrm{~mm} \mathrm{SVC}=7 \times 10^{5} \mathrm{~mm} / \mathrm{min}$ Angular Coherency: $85 \%$

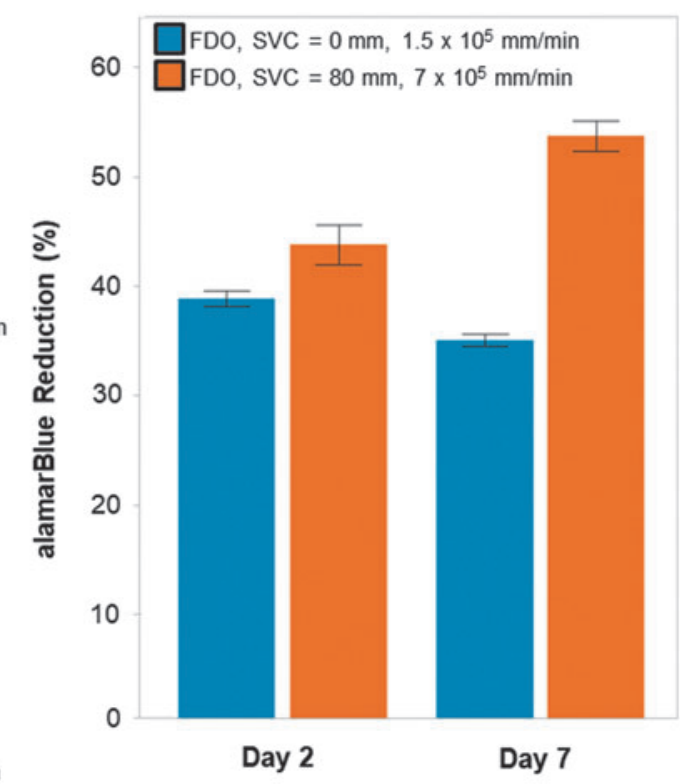

FIG. 3. Scaffolds support cell proliferation. Differences in $3 \mathrm{~T} 3$ cell viability and metabolic activity of scaffolds with contrasting fibrous morphologies fabricated with different combinations of $3 \mathrm{DMB}$ parameters indicated by (left) Live/Dead images at day 1 , and (right) $\%$ alamarBlue reduction at days 2 and 7. 


\section{Flexibility of Multiple Materials}
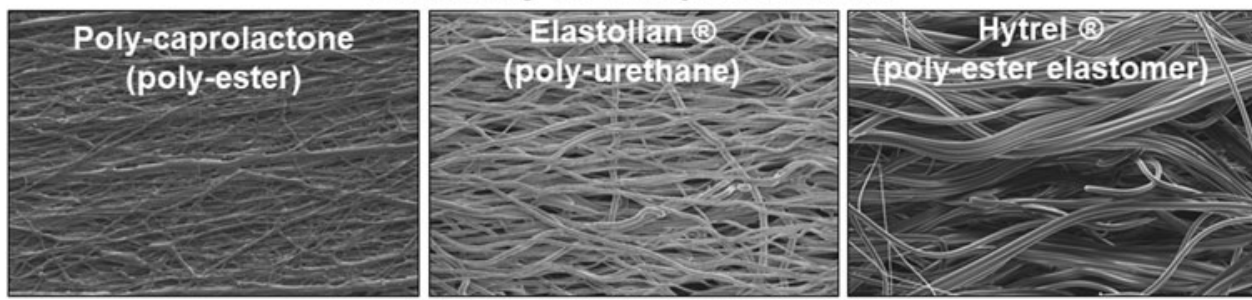

FIG. 4. 3DMP for distinct fiber materials, diameters, and orientation. The compatibility of multiple medical-grade thermoplastics has been demonstrated using this $3 \mathrm{DMB}$ process (top row). Control of fiber diameter and organization has also been demonstrated for these materials. For example, this process is capable of tuning PCL fiber diameter (middle row) and orientation (bottom row). The ability of 3DMB to tailor fiber macro- and microstructure is promising for repair strategies for tissues with highly specialized fibrous architectures.

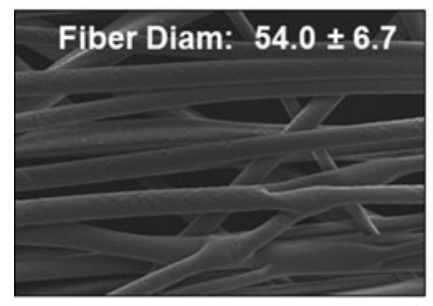

Control of Fiber Diameter

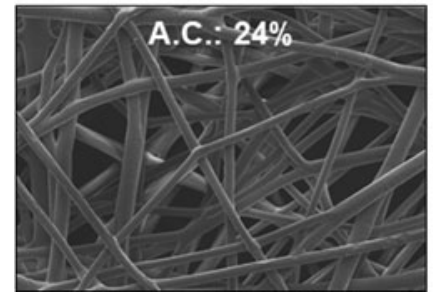

Control of Fiber Orientation
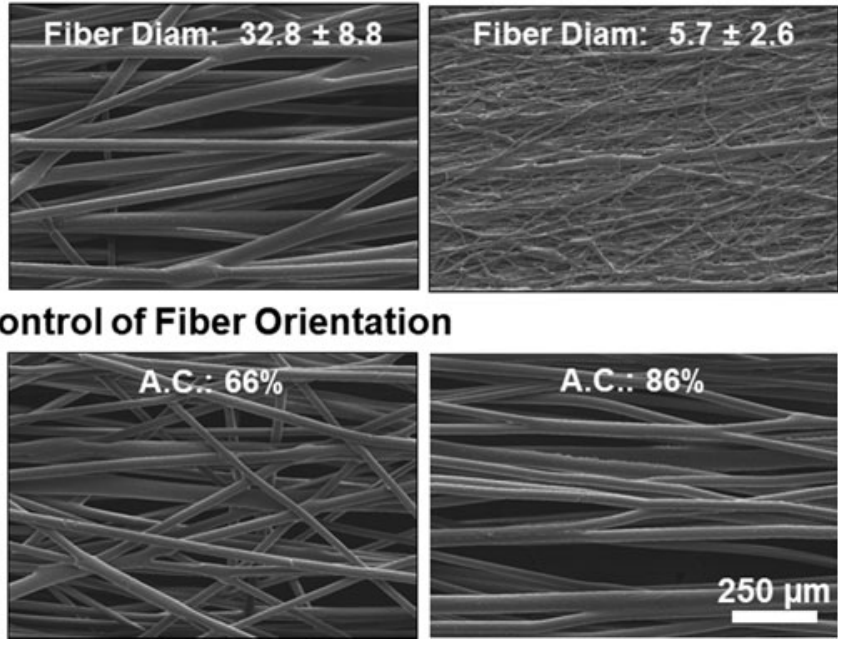

made from the same material (PCL), but with different fiber diameter, are shown in Figure 4 (middle row). In general, for PCL, mean fiber diameter could be varied from $\sim 60 \mu \mathrm{m}$ in diameter down to $\sim 5 \mu \mathrm{m}$. More specifically, the representative PCL samples have fiber sizes (mean \pm standard deviation) of $54.0 \pm 6.7 \mu \mathrm{m}, 32.8 \pm 8.8 \mu \mathrm{m}$, and $5.7 \pm 2.6 \mu \mathrm{m}$. This low variability $(\sim \pm 2-3 \mu \mathrm{m}$ at low diameters and $\sim 7-9 \mu \mathrm{m}$ at high diameters) provides for fine tuning of fibers in scaffolds to mimic natural fibers for specific applications or to probe scientific questions regarding specific ranges of fiber diameters. Importantly, this range of fibers represents a size scale difficult or impossible to achieve using large 3D printing systems and includes the upper range of fiber diameters achieved through electrospinning.

The 3DMB system also offers the ability to tune fiber orientation, using specific collectors moving at high rates of speed as in Figure 4 (bottom row). To demonstrate this capability, we fabricated scaffolds made from the same material (PCL) using the rotating drum collector (Fig. 1B). To control fiber orientation, the speed of the drum (SVC) was varied. At low speeds, more random fiber orientations were achieved. For example, in Figure 4 (lower left panel) an angular coherency of only $24 \%$ was observed. As drum speed increased, more oriented configurations were achieved (Fig. 4, lower center and right panels). More importantly, orientation could be controlled, allowing the creation of scaffolds with $66 \%$ or $86 \%$ of fibers aligned along the direction of motion depending on the speed used. Similar to the discussion of fiber size, this fine control of orientation will enable fabrication of more consistent and specialized scaffolds while also providing a methodology for characterizing the impact of slight variations in fiber orientation for a given application.
Scaffold example 1: device fabricated from two different kinds of fibers. The objective was to create a bioresorbable hernia plug for canine therapy. The starting shape is a cylinder, which the surgeon flips partially inside out to form an insertable plug (Fig. 5). After removal of extruded hernial tissue, the flipped end of the cylinder would be inserted into the defect. After cutting the material to release the flipped portion internal to the abdominal wall and to shorten the cylinder to the desired size on the external wall, the ends would be pulled in opposite directions and stapled into place. To accomplish this manipulation, the cylinder needs to be compressible, strong, and elastic.

The fabric used to make the initial cylinder was composed of a layer of PCL and a layer of PLA. The 3DMB was performed using a high speed of rotation for the collection drum, thereby creating a high orientation of the fibers in the resulting fabric (Fig. 5). PLA by itself made cylinders that were too brittle to roll, and PCL by itself did not provide the tensile strength required. Sequentially printing the PLA and PCL on the same cylinder produced two-layer cylinders that had both sufficient flexibility and strength. The high degree of orientation of fibers in the fabric provides the structure with the extra elasticity needed for ease of flipping one end of the cylinder inside out while wearing surgical gloves.

The prototypes were successfully implanted in dog cadavers (Fig. 5), demonstrating that the basic requirements for the mechanical behavior were met: the end of the cylinder could be flipped easily, and the fabric did not tear or fall apart while handled. What remains to be analyzed are the in vivo metrics such as degradation time, foreign body reaction, and healing rates. As the evaluation of clinical utility progresses, the degradation time of each polymer can be independently tuned to provide extended support as the healing progresses. 

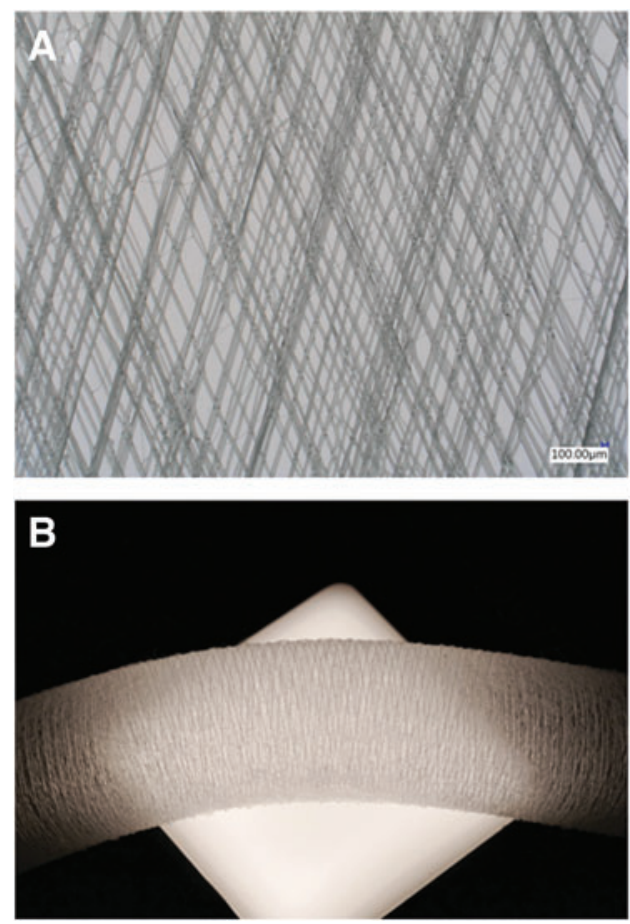
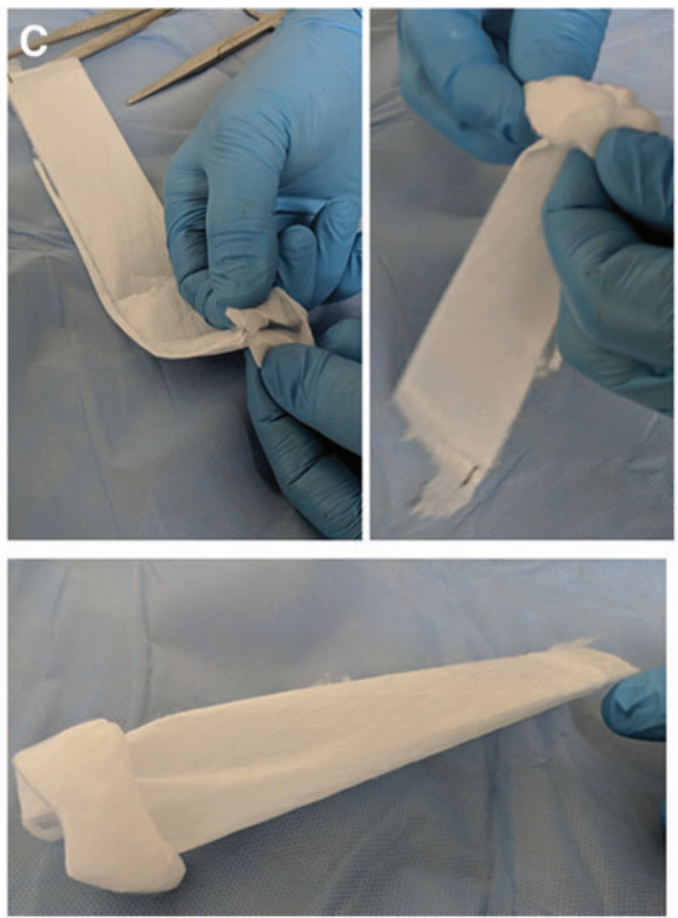
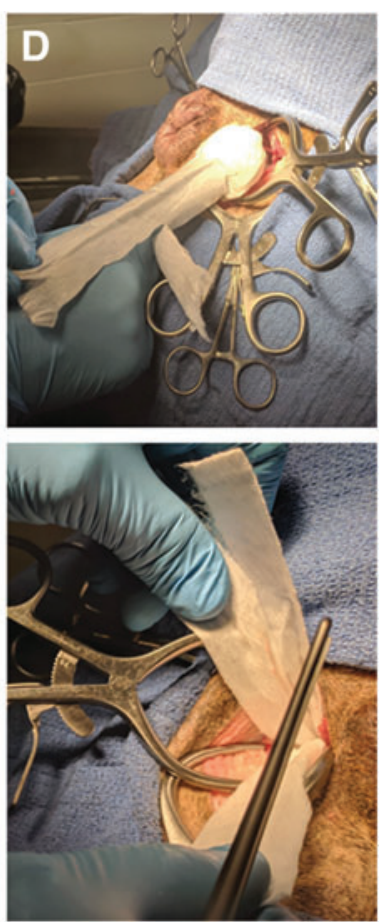

FIG. 5. Double layer scaffold for hernia repair device. (A) bilayer scaffold produced from a PCL layer and a PLA layer oriented at angles to each other. (B) Photograph showing the long-range extent of orientation of fibers in the two-layer PCL/ PLA hernia repair tube. (C) Roll-down method performed on the bilayer PCL + PLA mesh tube. (D). Insertion into canine cadaver for hernia repair. PLA, polylactic acid.

Scaffold example 2: scaffold with complex 3D architecture. The menisci within the knee joint are complex structures that transmit and distribute loads between the tibia and femur. In one plane, the menisci have a semilunar macroscale geometry (Fig. 6A, C). In another plane, the menisci have a wedge-shaped geometry. Such a complex geometry allows the menisci to be congruent to both the spherical shape of the femoral condyles and the planar shape of tibial plateau and allow effective force transmission (Fig. 6A). The collagen fiber bundles in the menisci are densely packed
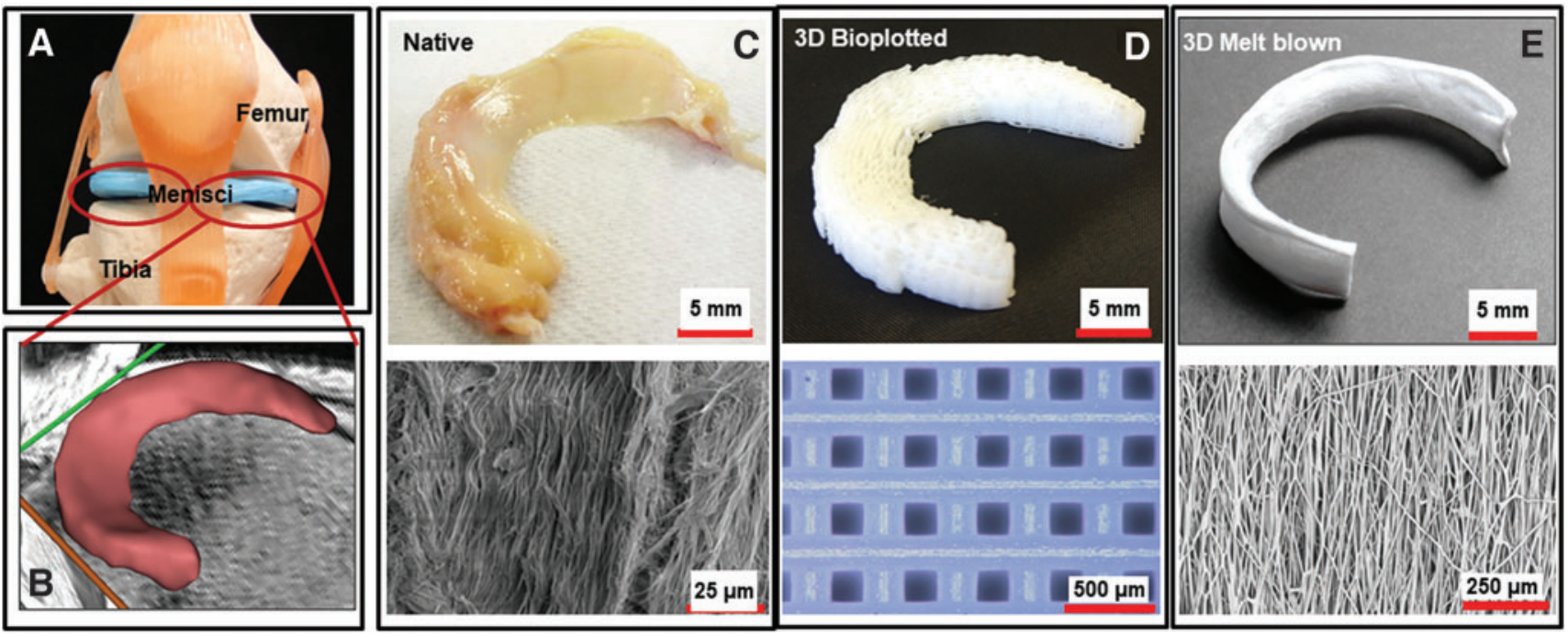

FIG. 6. Scaffold for meniscus replacement. (A) Menisci are cartilaginous tissues located between the tibia and femur to act as shock absorbers. Currently, surgical repair or allografts are used to treat meniscus injuries, but no FDA-approved replacement device exists in the United States. Different 3D printing approaches are being investigated to create tissueengineered alternatives. Once the patient-specific geometry is biomodeled from the patient's MRI scans (B), these 3D biomodels can be used in 3D printing to recapitulate the 3D shape. The macroscale shape and microscale fibrous microarchitecture of these scaffolds is shown for natural tissue (C), a 3D bioplotted PCL scaffold (D) and a 3DMB PCL scaffolds (E). FDA, Food and Drug Administration; MRI, magnetic resonance imaging. 
and mostly oriented in the circumferential direction, with the occasional radial "tie" fiber or sheet (Fig. 6C). This scaffold architecture is important for the meniscus to function under compression, tension, and shear. Thus, to engineer a scaffold to replace the meniscus, both the micro- and macrostructural features must be matched.

To match the macroscale geometry, patient-specific geometry can be modeled from a patient's MRI scans (Fig. 6B). In 3D bioplotting (extrusion-based 3D printing), this information is imported into software used to control a path of polymer deposition allowing excellent reconstruction of the 3D macroscale geometry (Fig. 6D). However, this process has fibers on the order of hundreds of microns and the resulting scaffold does not match the microstructure of the meniscus.

Using 3DMB, we matched the macroscale of the meniscus by using the patient-specific geometry to fabricate an inverse mold of the meniscus as shown schematically in Figure 1B. Then, we collected fibers within the void space of the collector while it was rotated as a high rate of speed, resulting in complete filling of the void space and a macroscale geometry similar to the meniscus. The 3DMB fibers were well-aligned along the principal direction of collector rotation (fiber diameter $=12 \mu \mathrm{m}$, angular coherency $=72 \%$ ), and the microarchitecture of this scaffold more closely resembles that of the native meniscus (Fig. 6E). Moreover, while the 3DP process takes $\sim 4 \mathrm{~h}$, the $3 \mathrm{DMB}$ process requires less than 15 min to make a scaffold for the same meniscus geometry.

Tradeoffs for achieving high-throughput scaffold production. Despite the advantages of this new system, some limitations and challenges exist that coincide with the focus on achieving high throughput. First, in 3DMB the focus of the process is on achieving high throughput and anatomically sized scaffolds with biologically relevant fiber sizes; thus the direct control over individual fiber deposition typical of EDP processes is not possible. Second, the higher throughputs in 3DMB necessitate larger volumes of polymers to operate the system. The typical volume of polymer raw material needed to operate the melt-blowing subsystem $(\sim 100 \mathrm{~g})$ is an order of magnitude higher than volumes needed for electrospinning or 3DP systems; note that this is only a disadvantage if scaffolds are to be produced one at a time Third, in its current form, the 3DMB system is suitable for processing only molten thermoplastics and not for biologics and other thermosensitive biomaterials. To process these other materials, the setup will have to be modified to replace the melt-blowing subsystem with a solution blowing apparatus. Finally, the 3DMB system is larger than a typical electrospinning or 3DP apparatus. As a result, the system cannot be placed inside a biosafety cabinet for aseptic processing. To make scaffolds for clinical studies, the system needs to be located in a GMP clean room.

\section{Conclusions}

The 3D fiber melt-blowing process introduced in this study has demonstrated the capacity to produce biocom- patible fibers with predefined sizes and spacing, combinations of fibers with different chemical and physical properties, and fiber deposition with orientation controlled in three dimensions. Unlike conventional melt-blowing systems, the $3 \mathrm{DMB}$ process is configured for efficient use with the relatively expensive polymers necessary for biomedical applications, decreasing the required amounts of material for processing while achieving high throughputs compared with 3D printing or electrospinning. The 3DMB technology has been demonstrated to make scaffolds composed of multiple fiber materials and organized into complex shapes, including those typical of human body parts.

The 3DMB process has been designed to be adaptable for inclusion of cells as well as biocompatible polymers. The air curtain around the fluid as it passes through each nozzle not only separates the fibers from each other during the blowing process, but also reduces the shear forces that a cell passing through the system would encounter. The remaining challenge to print cells simultaneously with the fibers is to demonstrate printing at $37^{\circ} \mathrm{C}$, preferably with biopolymers such as collagen or gelatin.

Early in vitro studies presented here have demonstrated the ability of PCL scaffolds made through 3DMB to support cell attachment and proliferation. In future, we will develop hybrid processing strategies to incorporate relevant growth factors (e.g., proteins, peptides) to enhance the bioactivity of scaffolds for specific tissue applications and investigate the functionality of scaffolds in vivo.

\section{Acknowledgment}

The AIF is a member of the North Carolina Research Triangle Nanotechnology Network (RTNN), a site in the National Nanotechnology Coordinated Infrastructure (NNCI).

\section{Disclosure Statement}

A patent application has been filed on the 3DMB system, but the intellectual property is not currently licensed (U.S. application 62821743: "Nonwoven Structures Comprising Oriented Meltblown Fibers and Processes for Making Them"). The authors have no financial interest that would constitute a conflict of interest.

\section{Funding Information}

This work was supported by the Game-Changing Research Incentive Program (GRIP) and the Nonwovens Institute at North Carolina State University. Electron microscopy was performed in part at the Analytical Instrumentation Facility (AIF) at North Carolina State University, which is supported by the State of North Carolina and the National Science Foundation (award no. ECCS-1542015).

\section{Supplementary Material}

Supplementary Table S1 


\section{References}

1. Mauck, R.L., Baker, B.M., Nerurkar, N.L., et al. Engineering on the straight and narrow: the mechanics of nanofibrous assemblies for fiber-reinforced tissue regeneration. Tissue Eng Part B Rev 15, 171, 2009.

2. Jenkins, T.L., and Little, D. Synthetic scaffolds for musculoskeletal tissue engineering: cellular responses to fiber parameters. NPJ Regen Med 4, 1, 2019.

3. Yang, F., Murugan, R., Ramakrishna, S., Wang, X., Ma, Y.X., and Wang, S. Fabrication of nano-structured porous PLLA scaffold intended for nerve tissue engineering. Biomaterials 5, 1891, 2004.

4. Xu, C.Y., Inai, R., Kotaki, M., and Ramakrishna, S. Aligned biodegradable nanofibrous structure: a potential scaffold for blood vessel engineering. Biomaterials 25, 877, 2004.

5. Kumbar, S.G., Nukavarapu, S.P., James, R., Nair, L.S., and Laurencin, C.T. Electrospun poly(lactic acid-co-glycolic acid) scaffolds for skin tissue engineering. Biomaterials 29 , 4100, 2008.

6. Li, M., Mondrinos, M.J., Chen, X., Gandhi, M.R., Ko, F.K., and Lelkes, P.I. Co-electrospun poly(lactide-co-glycolide), gelatin, and elastin blends for tissue engineering scaffolds. J Biomed Mater Res Part A 79A, 963, 2006.

7. Kobayashi, M., Lei, N.Y., Wang, Q., Wu, B.M., and Dunn, J.C.Y. Orthogonally oriented scaffolds with aligned fibers for engineering intestinal smooth muscle. Biomaterials 61, 75, 2015.

8. Burton, T.P., and Callanan, A. A non-woven path: electrospun poly(lactic acid) scaffolds for kidney tissue engineering. Tissue Eng Regen Med 15, 301, 2018.

9. Murugan, R., and Ramakrishna, S. Design strategies of tissue engineering scaffolds with controlled fiber orientation. Tissue Engineering 13, 1845, 2007.

10. Warren, P.B., Huebner, P., Spang, J.T., Shirwaiker, R.A., and Fisher, M.B. Engineering 3D-bioplotted scaffolds to induce aligned extracellular matrix deposition for musculoskeletal soft tissue replacement. Connect Tissue Res 58, 342, 2017.

11. Yoshimoto, H., Shin, Y.M., Terai, H., and Vacanti, J.P. A biodegradable nanofiber scaffold by electrospinning and its potential for bone tissue engineering. Biomaterials 24, 2077, 2003.

12. Badylak, S.F. The extracellular matrix as a scaffold for tissue reconstruction. Semin Cell Dev Biol 13, 377, 2002.

13. Frantz, C., Stewart, K.M., and Weaver, V.M. The extracellular matrix at a glance. J Cell Sci 123, 4195, 2010.

14. Nam, Y.S., and Park, T.G. Porous biodegradable polymeric scaffolds prepared by thermally induced phase separation. J Biomed Mater Res 47, 8, 1999.

15. Liu, X., and Ma, P.X. Phase separation, pore structure, and properties of nanofibrous gelatin scaffolds. Biomaterials 30, 4094, 2009.

16. Liao, C.-J., Chen, C.-F., Chen, J.-H., Chiang, S.-F., Lin, Y.-J., and Chang, K.-Y. Fabrication of porous biodegradable polymer scaffolds using a solvent merging/ particulate leaching method. J Biomed Mater Res 59, 676, 2002.

17. Sin, D.C., Miao, X., Liu, G., et al. Polyurethane (PU) scaffolds prepared by solvent casting/particulate leaching (SCPL) combined with centrifugation. Mater Sci Eng C 30, 78, 2010.
18. Nam, Y.S., Yoon, J.J., and Park, T.G. A novel fabrication method of macroporous biodegradable polymer scaffolds using gas foaming salt as a porogen additive. J Biomed Mater Res 53, 1, 2000.

19. Mondrinos, M.J., Koutzaki, S., Jiwanmall, E., et al. Engineering three-dimensional pulmonary tissue constructs. Tissue Eng 12, 717, 2006.

20. Dehghani, F., and Annabi, N. Engineering porous scaffolds using gas-based techniques. Curr Opin Biotechnol 22, 661, 2011.

21. Moshfeghian, A., Tillman, J., and Madihally, S.V. Characterization of emulsified chitosan-PLGA matrices formed using controlled-rate freezing and lyophilization technique. J Biomed Mater Res Part A 79A, 418, 2020.

22. Doshi, J., and Reneker, D.H. Electrospinning process and applications of electrospun fibers. J Electrostat 35, 151, 1995.

23. Chronakis, I.S. Novel nanocomposites and nanoceramics based on polymer nanofibers using electrospinning process-a review. J Mater Process Technol 167, 283, 2005.

24. Narayanan, L.K., Huebner, P., Fisher, M.B., Spang, J.T., Starly, B., and Shirwaiker, R.A. 3D-bioprinting of polylactic acid (PLA) nanofiber-alginate hydrogel bioink containing human adipose-derived stem cells. ACS Biomater Sci Eng 2, 1732, 2020.

25. Mellor, L.F., Huebner, P., Cai, S., et al. Fabrication and evaluation of electrospun, 3d-bioplotted, and combination of electrospun/3d-bioplotted scaffolds for tissue engineering applications. Biomed Res Int 2017, 6956794, 2017.

26. Szojka, A., Lalh, K., Andrews, S.H.J., Jomha, N.M., Osswald, M., and Adesida, A.B. Biomimetic 3D printed scaffolds for meniscus tissue engineering. Bioprinting $\mathbf{8}, 1$, 2017.

27. Suwantong, O. Biomedical applications of electrospun polycaprolactone fiber mats. Polym Adv Technol 27, 1264, 2016.

28. Warren, P.B., Davis, Z.G., and Fisher, M.B. Parametric control of fiber morphology and tensile mechanics in scaffolds with high aspect ratio geometry produced via melt electrowriting for musculoskeletal soft tissue engineering. J Mech Behav Biomed Mater 99, 153, 2019.

29. Wei, C., and Dong, J. Direct fabrication of high-resolution three-dimensional polymeric scaffolds using electrohydrodynamic hot jet plotting. J Micromech Microeng 23, 025017, 2013.

30. Tuin, S., Pourdeyhimi, B., and Loboa, E.G. Creating tissues from textiles: scalable nonwoven manufacturing techniques for fabrication of tissue engineering scaffolds. Biomed Mater 11, 015017, 2016.

31. Schuchard, K., Pourdeyhimi, B., Fisher, M., and Shirwaiker, R. Investigating process-structure relationships of 3D-melt blowing for tissue engineering applications. Proc 2019 IISE Annual Conference, 2019, Orlando, FL (paper ID 583734, in press).

32. Jenkins, T.L., Meehan, S., Pourdeyhimi, B., and Little, D. Meltblown polymer fabrics as candidate scaffolds for rotator cuff tendon tissue engineering. Tissue Eng A 23, 958, 2017.

33. Niu, H., Lin, T., and Wang X. Needleless electrospinning. I. A comparison of cylinder and disk nozzles. J Appl Polym Sci 114, 3524, 2009. 
34. Schuchard, K., Anderson, B., Grondin, P., Fisher, M.B., Pourdeyhimi, B., and Shirwaiker, R.A. Anisotropic scaffold fabrication using high-throughput 3D-melt blowing. International Conference on Biofabrication. Columbus, $\mathrm{OH}, 2019$ (abstract of presentation listed in session 13, pp. 39).

35. Sinha-Ray, S., Yarin, A.L., and Pourdeyhimi, B. Meltblowing: I-basic physical mechanisms and threadline model. J Appl Phys 108, 034912, 2010.

36. Majumdar, B., and Shambaugh, R.L. Air drag on filaments in the melt blowing process. J Rheol 34, 591, 1990.

37. Uyttendaele, M.A.J., and Shambaugh, R.L. Melt blowing: general equation development and experimental verification. AIChE J 36, 175, 1990.

38. Robert, M., and Shambaugh, L. A Macroscopic view of the melt-blowing process for producing microfibers. Ind Eng Chem Res 27, 2363, 1988.

39. Hassan, M.A., Yeom, B.Y., Wilkie, A., Pourdeyhimi, B., and Khan, S.A. Fabrication of nanofiber meltblown membranes and their filtration properties. J Membr Sci 427, 336, 2013.
40. Yarin, A.L., Sinha-Ray, S., and Pourdeyhimi, B. Meltblowing: multiple polymer jets and fiber-size distribution and lay-down patterns. Polymer (Guildf) 52, 2929, 2011.

Address correspondence to: Frances S. Ligler, DPhil, DSc Joint Department of Biomedical Engineering University of North Carolina at Chapel Hill and North Carolina State University EB3-Room 43071840 Entrepreneur Drive Raleigh, NC 27597-7115 USA

E-mail: fsligler@ncsu.edu

Received: April 20, 2020

Accepted: June 10, 2020

Online Publication Date: July 15, 2020 